\title{
Secondary Payload Opportunities on NASA's Space Launch System (SLS) Enable Science and Deep Space Exploration
}

\author{
Jody Singer ${ }^{1}$ \\ National Aeronautics and Space Administration, Marshall Space Flight Center, Huntsville, Alabama 35812 \\ Joseph Pelfrey ${ }^{2}$ \\ National Aeronautics and Space Administration, Marshall Space Flight Center, Huntsville, Alabama 35812 \\ George Norris ${ }^{3}$ \\ National Aeronautics and Space Administration, Marshall Space Flight Center, Huntsville, Alabama 35812
}

\section{Introduction}

For the first time in almost 40 years, a NASA human-rated launch vehicle has completed its Critical Design Review (CDR). With this milestone, NASA's Space Launch System (SLS) and Orion spacecraft are on the path to launch a new era of deep space exploration. This first launch of SLS and the Orion Spacecraft is planned no later than November 2018 and will fly along a trans-lunar trajectory, testing the performance of the SLS and Orion systems for future missions. NASA is making investments to expand the science and exploration capability of the SLS by developing the capability to deploy small satellites during the trans-lunar phase of the mission trajectory. Exploration Mission 1 (EM-1) will include thirteen 6U Cubesat small satellites to be deployed beyond low earth orbit. By providing an earthescape trajectory, opportunities are created for the advancement of small satellite subsystems, including deep space communications and in-space propulsion. This SLS capability also creates low-cost options for addressing existing Agency strategic knowledge gaps and affordable science missions. A new approach to payload integration and mission assurance is needed to ensure safety of the vehicle, while also maintaining reasonable costs for the small payload developer teams. SLS EM-1 will provide the framework and serve as a test flight, not only for vehicle systems, but also payload accommodations, ground processing, and on-orbit operations. Through developing the requirements and integration processes for EM-1, NASA is outlining the framework for the evolved configuration of secondary payloads on SLS Block upgrades. The lessons learned from the EM-1 mission will be applied to processes and products developed for future block upgrades. In the heavy-lift configuration of SLS, payload accommodations will increase for secondary opportunities including small satellites larger than the traditional Cubesat class payload. The payload mission concept of operations, proposed payload capacity of SLS, and the payload requirements for launch and deployment will be described to provide potential payload users an understanding of this unique exploration capability.

\section{Hardware/System Overview}

\section{A. Space Launch System}

The SLS is the new heavy launch system for NASA. The SLS configuration for EM-1 is considered Block 1, the first configuration of the SLS evolution plan. The Shuttle-derived design takes advantage of resources established for the Space Shuttle, including the workforce, tooling, manufacturing processes, supply chain, transportation logistics, launch infrastructure, and LOX/LH2 propellant infrastructure. An overview of the initial SLS Block 1 configuration that will first fly with the Orion in 2018 is shown in Fig.1. The SLS enables many aspects of the NASA core capabilities in addition to human exploration initiatives. These include the reduction in mission duration, increased mass margins, reductions in total spacecraft complexity, and significant increases in payload volume.

\footnotetext{
${ }^{1}$ Marshall Space Flight Center Deputy Director, Office of the Director, Mail Code DA01, jody.singer@nasa.gov

${ }^{2}$ Manager, Exploration and Space Transportation Development Office Mail Code FP30, joseph.pelfrey@nasa.gov

${ }^{3}$ Exploration Payloads Team Lead, Exploration and Space Transportation Development Office Mail Code FP30, george.norris-1@nasa.gov
} 

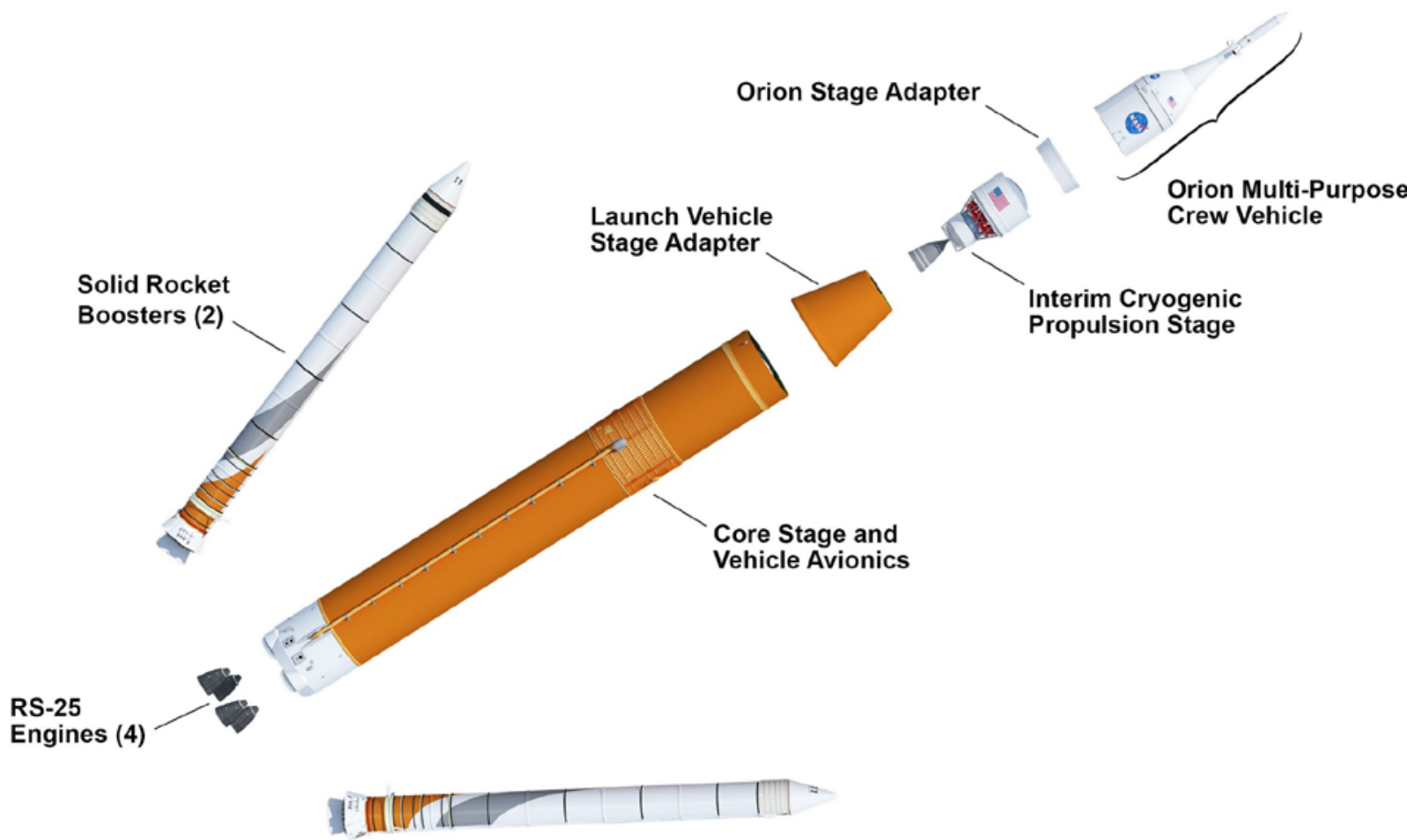

Fig. 1 SLS Block 1 70t Initial Configuration: The Orion Stage Adaptor will hold 13 6u cubesats to be launched into deep space.

The secondary payload initiative for EM-1 takes advantage of several of these capabilities and enables new opportunities for small spacecraft developers. By utilizing planned unoccupied volume within the upper stage adapter ring, the Orion Stage Adapter (OSA), increased mission science and technology missions can be accommodated.

SLS Block 1 is capable of deploying 70 metric tons of payload into low Earth orbit. The characteristic energy $\left(\mathrm{C}_{3}\right)$ curve for SLS is provided in Fig. 2, illustrating SLS's evolved thrust capabilities.

SLS will launch from Kennedy Space Center with main engine start, booster ignition, and primary ascent operations up to the point of upper stage separation. After upper stage separation and execution of the trans-lunar orbital injection burn, the Orion spacecraft will separate from the upper stage, and Orion will begin autonomous operations. The upper stage will then complete a mission disposal maneuver that will slow the upper stage and attached interfaces, including the OSA, and thus place the expired motor on a non-contact with Orion, trans-lunar heliocentric disposal trajectory. The upper stage system will remain on battery power for several hours after the completion of the Orion separation and disposal trajectory maneuvers. Deployments of the secondary payloads will commence after sufficient separation of the Orion spacecraft to the upper stage vehicle to minimize any possible contact of the deployed cubesats to Orion. Currently this is estimated to require approximately 4 hours. The allowed deployment window for the cubesats will be from the time the upper stage disposal maneuvers are complete to up to 10 days after launch. The upper stage will fly past the moon at a perigee of approximately $100 \mathrm{~km}$, and this closest approach will occur about 5 days after launch. The limiting factor for the latest deployment time is the available power in the sequencer system (described in subsequent section).

\section{B. Payload Accomodations}

Secondary payloads on EM-1 will be launched in the Orion Stage Adapter (OSA). Payload dispensers will be mounted on specially designed brackets, each attached to the interior wall of the OSA as shown in Fig. 3. For the EM1 mission, a total of fourteen brackets will be installed, allowing for thirteen payload locations. The final location will be used for mounting an avionics unit, which will include a battery and sequencer for executing the mission deployment sequence. 


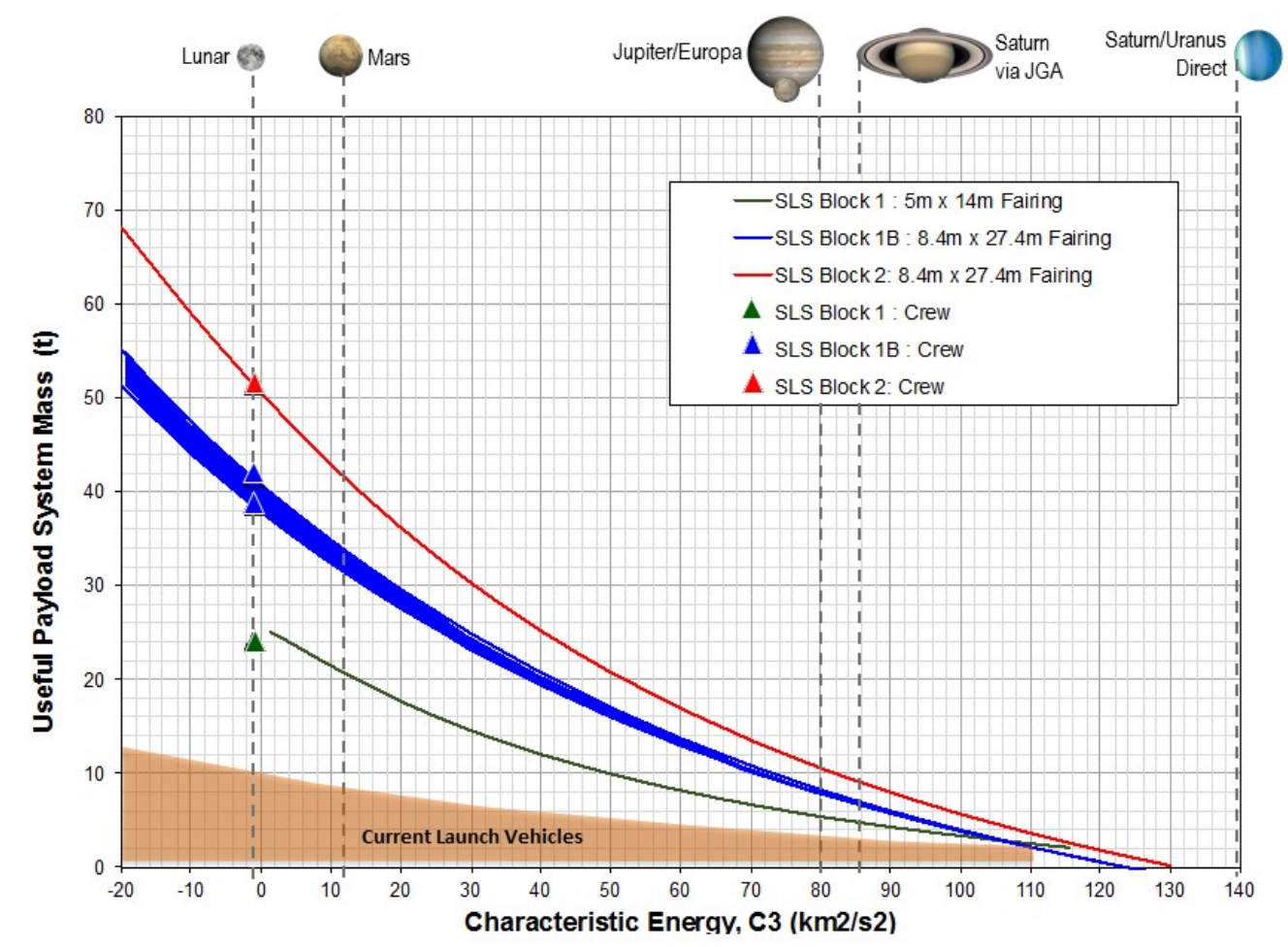

Fig. 2 SLS Net-Payload System Mass-Earth Escape: The SLS characteristic energy $\left(C_{3}\right)$ curve illustrates SLS's evolved thrust capabilities. SLS Block 1 is capable of deploying 70 metric tons of payload into low Earth orbit.

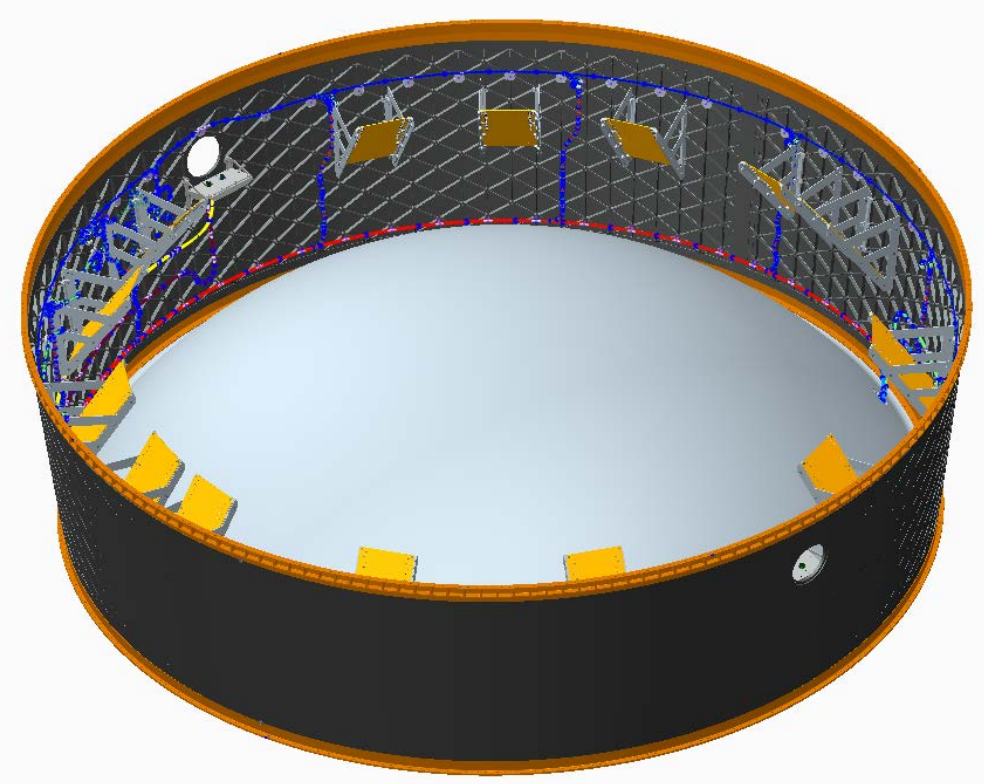

Fig. 3 Payload Accomodations within the Orion Stage Adaptor: Payload dispensers, mounted on specially designed brackets and attached to the interior wall of the OSA, allow for thirteen payload locations. The final location will hold an avionics unit. 
Each bracket is designed to hold the volume equivalent of $6 \mathrm{U}$ and $12 \mathrm{U}$ dispensers. The current design baseline for EM-1 is for payloads to be compatible with $6 \mathrm{U}$ class dispensers. Payloads in $6 \mathrm{U}$ class will be limited to $14 \mathrm{~kg}$ maximum mass. Detailed physical accommodations are documented in Fig. 4 and Table 1.

The avionics unit will interface with each deployer through cables mounted in the OSA. Payloads will remain powered off until the sequencer transmits the deployment signal to each dispenser, and the payload is released. Payloads will exit the dispenser at an approximate rate of $1.2 \mathrm{~m} / \mathrm{sec}$, with deployments separated by a minimum of 5 seconds. No other payload services are currently planned for EM-1.

The OSA on which the EM-1 secondary payloads will be mounted is unique to SLS Block 1 configuration. Current plans for the second launch, Exploration Mission-2, include an upgraded, more powerful Exploration Upper Stage in the Block 1B configuration. The SLS program is currently reviewing possibilities for accommodations for secondary and co-manifested payloads on this configuration of the vehicle. CubeSatclass payload size could increase from the $6 \mathrm{U}$ up to $27 \mathrm{U}$, and the upgraded vehicle also presents the potential for flying larger payloads on dedicated adapters for a total of $300 \mathrm{~kg}$.

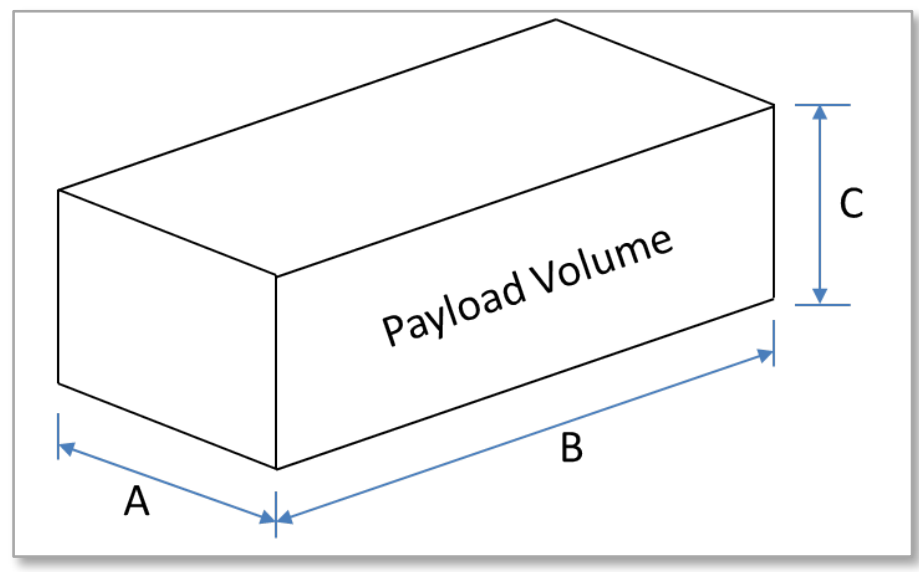

Fig. 4 Payload volume dimension

\begin{tabular}{|c|c|c|c|c|c|c|}
\hline \multirow{2}{*}{ Deployer } & \multicolumn{2}{|c|}{ A } & \multicolumn{2}{|c|}{ B } & \multicolumn{2}{|c|}{ C } \\
\cline { 2 - 7 } & in. & mm & in. & mm & in. & mm \\
\hline $\mathbf{6 U}$ & 9.43 & 239.4 & 14.4 & 366 & 4.6 & 116 \\
\hline $\mathbf{1 2 U}$ & 9.43 & 239.4 & 14.4 & 366 & 9.0 & 229 \\
\hline
\end{tabular}

Table 1 Payload maximum dimensions

\section{Ground Systems}

The Ground Systems Development and Operations (GSDO) program at the Kennedy Space Center (KSC) will perform SLS ground processing. Payloads will be fully integrated into their dispenser at the time of delivery to GSDO. Once delivered, integrated payloads will be installed into the OSA, prior to stacking operations with the Orion system. Prior to roll-out to the pad, battery charging for the avionics unit and each payload containing approved batteries will occur at the Vehicle Assembly Building $(\mathrm{VAB})$.

\section{Manifest Process}

Several NASA Mission Directorates have developed programs for the competition, selection, and development of EM-1 payloads that support Directorate priorities. Specific plans for payloads supported by these Directorates are explained in subsequent sections. There were several steps toward final determination of the planned manifest for the first mission. In the summer of 2015, each supporting mission directorate, the program directors for SLS, Orion, GSDO, and the Human Exploration and Operations senior leadership met to determine the number and allocation of payloads to be flown. Using this initial manifest, additional integrated safety hazard analysis and mass allocation reviews will be completed. Critical Design Review for the mission was completed in 2015, and a final mass allocation for the EM-1 secondary payload complement was determined. With these assessments and allocations made, a total manifest of 13 payloads was presented to the EM-1 Manifest Board for concurrence. The final manifest plan was presented to the Flight Planning Board in January of 2016 for baseline.

\section{Mission Integration}

The SLS Program will perform all mission and payload integration for the baseline vehicle manifest. The mission integration process defined in this section has been developed to ensure safety and mission success, while reducing the amount of data required from the payload developers. 
The integration process is designed to support the payload requirements as well as the requirements of the launch vehicle and ground systems. The typical integration process encompasses the entire cycle of payload integration activities including analytical and physical integration.

The Secondary Payloads Mission Integration Team (SPMIT) will be responsible for serving as liaison to the secondary payload community and facilitating end-to-end payload mission planning, integration, and execution. The mission integration team, in conjunction with the engineering and launch facility integration teams, will support secondary payload manifest planning, coordination, and end-to-end payload integration.

Each payload, once identified, will be assigned a Secondary Payload Integration Manager (SPIM) to assist the payload through the entire integration process. The SPIM will serve as the single point interface to coordinate all mission-specific integration activities for the assigned payload. The SPIM will be responsible for developing a payload-unique integration schedule to define the documentation and analysis required from the payloads. The SPIM will also capture payload requirements, engineering interfaces, and required verification through the payload-specific Interface Control Document (ICD).

The SPIM will coordinate the payload's requirements to all of the functional organizations involved in the mission. As the responsible point of contact, the SPIM will ensure that payload integration is accomplished by coordinating with these teams throughout the process.

Payload development schedules will vary based on many factors including hardware availability, complexity, resource profiles, etc. The payload life cycle is depicted in Fig. 5 and can be defined in four phases of integration:

1. Strategic:Early payload development occurs in the strategic timeframe and includes payload design and schedule development to ensure that an SLS compatible payload is built. The payload questionnaire is also submitted to support the manifest process, as well as early compatibility assessments.

2. Tactical: Focused mission integration is performed in the tactical phase of payload integration. During this phase, requirements and interfaces are finalized, verification is mostly completed, and the initial Certificate of Flight Readiness (CoFR) is developed.

3. Physical: This phase begins with the payload's on-dock arrival at the launch site and includes all ground processing, vehicle stacking, and integration. Final verifications are submitted, and the CoFR process is completed with closure of all remaining open work.

4. Operations: This phase begins with launch, through the end of payload operations. Post flight reporting and anomaly assessments will be conducted, as required.

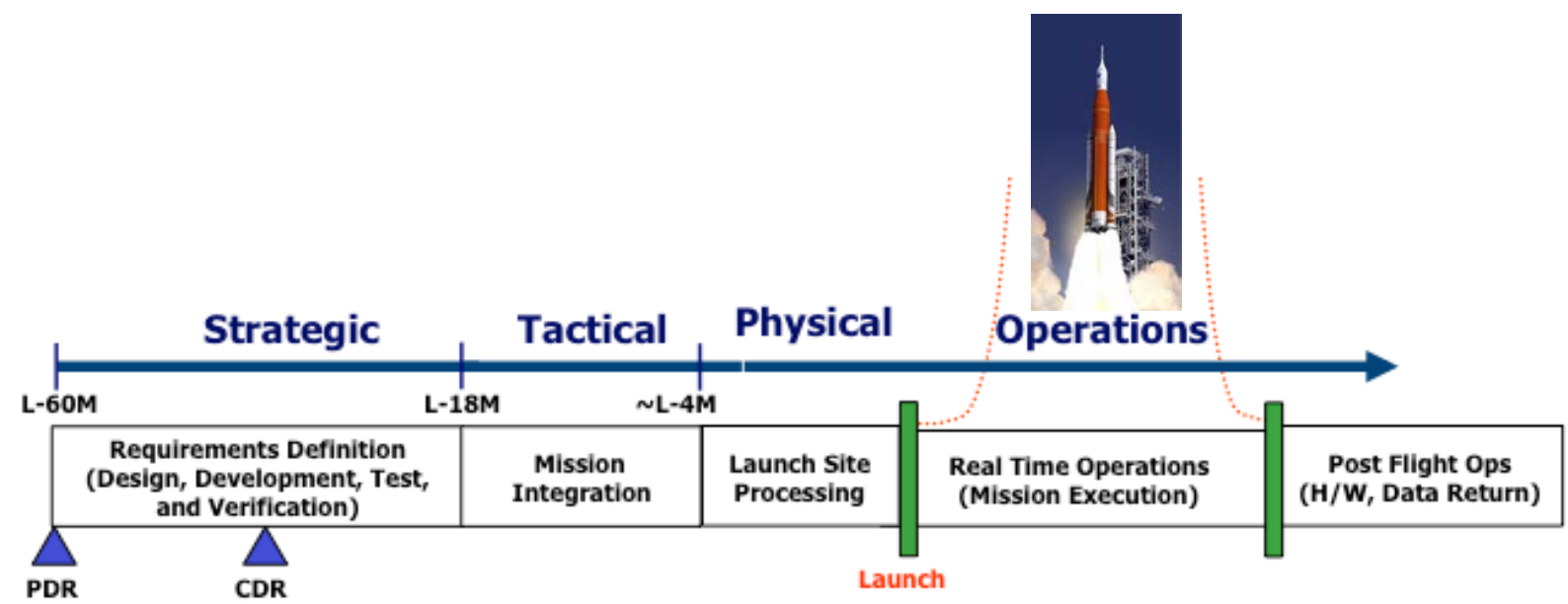

Fig. 5 Four Phases of the Payload Intergration Process

The typical SLS mission integration process begins at L-24 months, but can be tailored to meet payload needs within the constraints of the overall mission planning. The SPMIT may engage during the strategic phase to ensure early design requirements reflect the expected interfaces and environments to be verified during the mission integration process. The process will begin once a potential payload is identified and a SPIM is assigned. The payload will be provided with the questionnaire to return to the SPIM, and normal and regular communication/coordination will commence.

Throughout the mission integration phases, various reviews, and milestones will require payload developer participation and coordination with the mission integration team. The major milestones for each integration phase are outlined in Fig. 6. These include payload Preliminary Design Review (PDR), Critical Design Review (CDR), Safety 


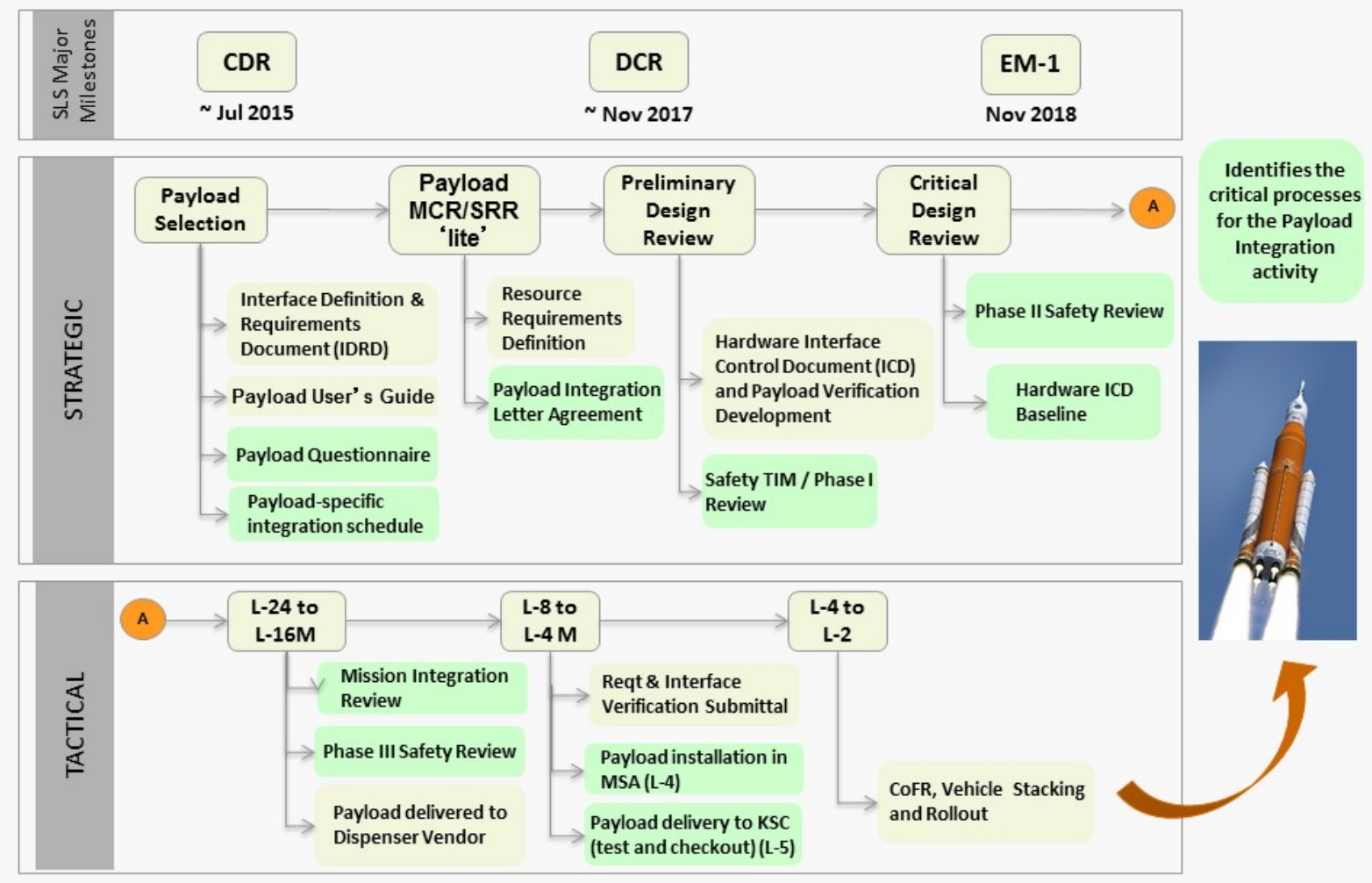

Fig. 6 Major milestones of the payload integration life cycle include Preliminary Design Review, Critical Design Review, Safety Reviews, major documentation baseline, and physical integration.

Reviews, major documentation baseline, and physical integration. Detailed mission-specific milestones and reviews will be documented in the payload integration schedule once a flight manifest is baselined.

To facilitate early integration and communication, payloads identified for SLS missions will be provided a payload questionnaire to provide NASA with pertinent data. This data will be used to evaluate manifest opportunities for primary and secondary payloads. The questionnaire will also be used as a starting point for developing the payloadunique ICD and other integration documentation.

The Interface Control Document (ICD) defines the payload-to-vehicle and payload-to-ground system interfaces. The ICD will be derived from the Interface Definition and Requirements Document (IDRD) and will identify the specific technical and functional requirements that apply to the payload design. Mission-specific or payload-specific requirements will also be captured in the ICD. The PIM is responsible for developing the payload-unique ICD for each payload assigned to a mission.

Based on the set of requirements defined in the ICD, the applicable verification data required will be documented to ensure payload compatibility. The IDRD will define the verification data sheets for each requirement, including required data, success parameters and deliverable schedule. Once the payload-specific requirements are baselined, the PIM will use the deliverables captured in the IDRD to document the plan for verification in the payload integration schedule. As the payload hardware development is completed, required verification data will be submitted to the mission integration and engineering integration teams for review and approval.

For each mission, the Secondary Payload team will develop mission-specific analyses to verify compatibility with the launch vehicle, ground systems, data systems, and overall mission requirements. Required mission-specific analysis will be identified with the required interfaces, in the payload-unique applicability matrix listed in the payload's ICD. Payload-unique mission analysis will also be documented in the ICD, with the appropriate analysis parameters captured in an appendix. It is expected that test data will only be required to satisfy vibration, shock, mass, center of gravity, and electro-magnetic interface requirements. 
As part of the overall vehicle flight readiness process, payloads will participate in the Certificate of Flight Readiness (CoFR) process. The CoFR process is established to ensure an integrated flight vehicle has met requirements at all levels and is safe to fly. The payload CoFR process certifies flight readiness of payloads by all organizations involved in payload development, integration, launch, and on-orbit operation. The payloads CoFR process is defined by the SLS office and will be documented in the Certificate of Flight Readiness Plan.

The CoFR process starts approximately 9 months prior to launch. All organizations supporting the mission complete a CoFR endorsement checklist that identifies the open work remaining to complete the readiness process. Open work will be tracked until closure to ensure all requirements are met. Final CoFR approval occurs at the SLS Flight Readiness Review at L-2 weeks.

\section{Safety and Mission Assurance}

Payloads manifested on SLS will "do no harm" to the vehicle or crew and maintain the appropriate controls for critical or catastrophic hazards. Safety requirements will be documented in the SLSP EM-1 Payload Safety Requirements for Secondary Payload Hardware document for inclusion in payload designs. Payload developers will be required to submit safety data packages for each safety review identified in the SLSP EM-1 Secondary Payload Safety Review Process document. Typically, the Safety Technical Interchange Meeting (TIM) will outline the payload design and initial hazard identification. In the Phase I review, the preliminary payload design is reported. During the Phase II review, the final payload design is reported, including hazard controls. Ideally, all hazard reports are submitted for approval during this review. The Phase III review will evaluate the payload hazard verification and any final changes to the payload design and controls. All safety verification will require closure prior to the shipment of the payload to KSC for integration into the vehicle.

\section{Ground Processing}

Payloads will be turned over to GSDO fully integrated in their deployer, ready for installation in the OSA at approximately L-6 months. GSDO will install the integrated deployers onto the OSA brackets and mate all required connections for deployment signals. Payloads will not be accessible once stacking operations begin. For EM-1, additional tests and pad stay time is required to fully check out the vehicle configuration. Due to this "first flight" test activity, the vehicle will remain at the launch pad for up to two months, which will increase payload exposure to documented natural environments. Payload should consider this additional time into their design requirements for materials selection and battery life.

\section{Operations}

Secondary payloads on SLS will remain powered off during the ascent phase of the launch vehicle, through separation of the Orion spacecraft. Once separation is confirmed, the ICPS will send a discrete signal to the SPDS avionics to activate. The schedule for deployments will be loaded as a skit prior to vehicle stacking. No real-time commanding or telemetry is available; therefore payloads will be deployed automatically through the pre-determined mission timeline sequence.

Payloads will have opportunity to deploy beginning after the ICPS disposal sequence is complete (approximately $\mathrm{T}+4$ hours) up to 10 days from launch. All deployments will be completed before avionics batteries are expended. Fig. 7 provides an overview of the mission profile.

Once deployed, payloads will be required to wait 15 seconds before deploying antennas, solar panels, sails, etc. to ensure adequate clearance from ICPS. Payload communications following deployment will be the responsibility of the payload project, with no resources being provided by SLS.

\section{Payload Users}

\section{A. Human Exploration and Operations Mission Directorate Advanced Exploration Systems}

The Human Exploration and Operations Mission Directorate Advanced Exploration Systems (AES) Division was allocated five payload opportunities on the EM-1 mission. AES selected the first three payloads to fly on EM-1 at the same time the capability for accommodating Secondary Payloads on the SLS was being developed.

Near Earth Asteroid (NEA) Scout is a 6U cubesat designed to rendezvous and characterize a candidate NEA. A solar sail, another innovation to be demonstrated in the cubesat class, will provide propulsion. 


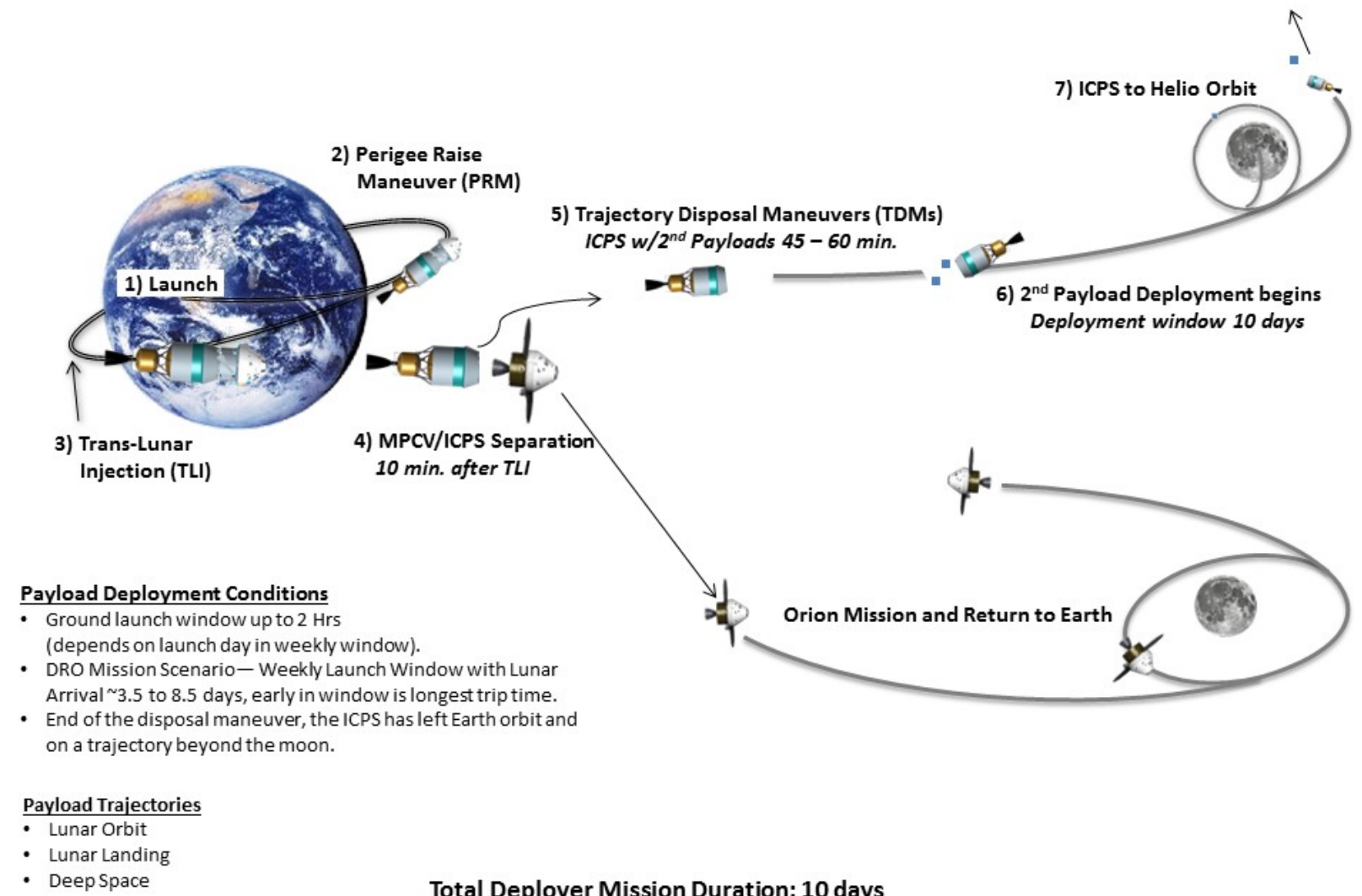

Fig. 7 Overview of Mission Profile: Secondary payloads remain powered off during launch vehicle ascent phase through Orion's separation of the spacecraft. Payloads will deploy based on a previously loaded schedule. Deployment opportunity begins at completion of ICPS disposal sequence ( T+4 hrs) up to 10 days from launch.

Lunar Flashlight is the second AES payload planned for manifest on EM-1. It will use a green propellant system and will search for potential ice deposits in the Moon's permanently shadowed craters. Pulsed lasars will be used to illuminate the surface. Surface reflection will be measured by a spectrometer to distinguish water ices from regolith.

The third payload being developed by AES is BioSentinel. The payload is a yeast radiation biosensor, planned to measure the effects of space radiation on deoxyribonucleic acid, or DNA. This will be accomplished by entering into a heliocentric orbit, outside of the Van Allen belts to expose the payload to a deep space radiation environment.

Two additional payloads were selected for the EM-1 mission by AES from the Next Space Technologies for Exploration Partnerships (NextSTEP) Broad Agency Announcement. The payloads selected are Lunar Icecube, a collaboration with Morehead State University, and Skyfire, a partnership with Lockheed Martin.

Lunar Icecube will prospect for water in ice, liquid, and vapor forms as well as other lunar volatiles from a lowperigee, highly inclined lunar orbit using a compact Infrared spectrometer.

Skyfire is a technology demonstration mission that will perform a lunar flyby, collecting spectroscopy, and thermography data to address questions related to surface characterization, remote sensing, and site selection.

\section{B. Space Technology Mission Directorate}

NASA's Space Technology Mission Directorate (STMD) was allocated three payload opportunities on the EM-1 mission. NASA's STMD is innovating, developing, testing, and flying hardware for use in NASA's future missions through the Centennial Challenges Program. The Centennial Challenges Program is NASA's flagship program for technology prize competitions (www.nasa.gov/challenges). The program directly engages the public, academia, and industry in open prize competitions to stimulate innovation in technologies that have benefit to NASA and the nation. STMD has released the CubeSat Lunar Challenge to foster innovations in small spacecraft propulsion and communications. There are two concurrent In-Space Competitions, the Lunar Derby and the Deep Space Derby. In 
the lunar Derby, prizes will be awarded for successfully achieving lunar orbit, downlinking the largest volume of error-free data, and surviving the longest. In the Deep Space Derby ( $>4$ million $\mathrm{km}$ ), there are prizes awarded for farthest data transmission distance, largest volume of error-free data, and longest duration of operability.

Potential candidates for the three STMD opportunities on the EM-1 mission will compete in a series of four Ground Tournaments before final selection is made in March of 2017.

\section{Science Mission Directorate}

The NASA Science Mission Directorate (SMD) was allocated two payload opportunities on the EM-1 mission. The NASA SMD issued an amendment to its annual Announcement of Opportunity (AO) in the Research Opportunities in Space and Earth Sciences-2014 (ROSES-2014) Solicitation NNH14ZDA001N-HTIDS Heliophysics Technology and Instrument Development for Science. Within this Amendment was the request for Cubesat proposals specific to the Exploration Mission 1 launch opportunity focusing on the Heliophysics science enabled through the unique deployment location and trajectory afforded though the planned EM-1 mission. The Cubesat Mission to Study Solar Particles (CuSP) payload was selected under this AO. CuSP will study the sources and acceleration mechanisms of solar and IP particles in near-Earth orbit, support space weather research by determining proton radiation levels during Solar Energetic Particle (SEP) events and identifying suprathermal properties that could help predict geomagnetic storms.

A Small Innovative Missions for Planetary Exploration (SIMPLEx) NASA Research Announcement (NRA) was also released as part of the ROSES-2014 AO. The LunaH-Map payload was selected from this NRA. The LunaH-Map objectives are to understand the quantity of H-bearing materials in lunar cold traps $(\sim 10 \mathrm{~km})$, determine the concentration of H-bearing materials with $1 \mathrm{~m}$ depth, and constrain the vertical distribution of H-bearing materials.

\section{International Partner Collaborations}

The final three payload opportunities for the EM-1 mission were allocated for NASA's international space agency counterparts. The flight opportunities are intended to benefit the international space agency and NASA as well as further the collective space exploration goals. A joint process with NASA and the international partners was employed to review, evaluate, and recommend the payloads to fly on EM-1. From that joint process three payloads were chosen: ArgoMoon, EQUilibriUm Lunar-Earth point 6U Spacecraft (EQUULEUS), and Outstanding MOon exploration TEchnologies demonstrated by NAno Semi-Hard Impactor (OMOTENASHI).

ArgoMoon is sponsored by European Space Agency/Italian Space Agency (ESA/ASI) and will fly-along with the ICPS on it's disposal trajectory. The primary objectives are to perform proximity operations with the ICPS postdisposal, take external imagery of engineering and historical significance, and perform an optical communications demonstration.

The EQUULEUS spacecraft sponsored by Japan Aerospace Exploration Agency (JAXA) will fly to a libration orbit around the Earth-Moon L2 point and demonstrate trajectory control techniques within the Sun-Earth-Moon region for the first time by a nano spacecraft. The mission will also contribute to the future human exploration scenario by understanding the radiation environment in geospace and deep space, characterizing the flux of impacting meteors on the far side of the moon, and demonstrating the future deep space exploration scenario using the "deep space port" at Lagrange points.

OMOTENASHI mission, sponsored by JAXA will land the smallest lunar lander to date on the lunar surface to demonstrate the feasibility of the hardware for distributed cooperative exploration system. Small landers will enable multi-point exploration, which is complimentary with large-scale human exploration. Once on the lunar surface, the OMOTENASHI spacecraft will observe the radiation and soil environments of the lunar surface by active radiation measurements and soil shear measurements.

\section{Conclusion}

NASA's Space Launch System (SLS) will provide unprecedented capability to further advances in science and exploration. The capability to deploy small satellites allows SLS to utilize excess capability on the planned exploration missions. With the planned mission trajectories, small satellite payload developers will have an opportunity to operate in deep space, a capability not realized to this point. As the SLS vehicle evolves its configuration and becomes more capable, the opportunities for Secondary Payloads of different types and sizes will increase.

\section{References}

1“SLS Mission Planners Program Mission Planners Guide, Version 2,” SLS-MNL-202, Sept. 22, 2015 